\title{
Classification of Current Routing Protocols for Ad Hoc Networks - A Review
}

\author{
Beigh Bilal Maqbool \\ Research Scholar \\ University of Kashmir (India)
}

\author{
Prof.M.A.Peer \\ Chairman BOPEE \\ J\&K (India)
}

\begin{abstract}
An ad hoc wireless network is a collection of two or more devices or nodes or terminals with wireless communications and networking capability that communicate with each other without the aid of any centralized administrator also the wireless nodes that can dynamically form a network to exchange information without using any existing fixed network infrastructure. And it's an autonomous system in which mobile hosts connected by wireless links are free to be dynamically and some time act as routers at the same time. In order to facilitate communication within the network, a routing protocol is used to discover routes between nodes. The primary goal of such an ad hoc network routing protocol is correct and efficient route establishment between a pair of nodes so that messages may be delivered in a timely manner. Route construction should be done with a minimum of overhead and bandwidth consumption. This article examines routing protocols for ad hoc networks and classify these protocols based on a set of parameters. The article provides an overview of different protocols by presenting their characteristics and functionality, and then provides a classification of these different routing protocols available for the transmission in ad hoc networks.
\end{abstract}

Key Words: Adhoc, Wireless, Protocols, Routing.

\section{INTRODUCTION}

Wireless network has become very popular in the computing industry. There are two types of wireless networks, The first is infrastructured network and second is infrastructure less network commonly known as ADHOC network. The first type of network consists of fixed and wired gateways. While as second type is a multihop wireless network and have no pre-defined infrastructure. The nodes in adhoc networks are dynamic in nature i.e they are capable of moving and are connected in an arbitrary fashion with each other. The adhoc networks are widely used in many civilian forums, military, business and emergency etc. For example, In civilian forum we use it in electronic classrooms, convention centres, construction sites and special events like live concerts and festivals etc.[16,13] Also some other areas of adhoc networks where it is used are participating in an interactive lectures, business associates sharing, soldiers relaying information about the situation awareness in a battlefield.

In this paper, We will discuss the current routing techniques/protocols and the classify them according to some certain set of parameters and characteristics, Which will give us an idea of designing some new technique/protocol in future.

\section{DESIRABLE PROPERTIES OF} AD-HOC ROUTING PROTOCOLS

The properties that are desirable in Ad-Hoc Routing protocols are

Distributed operation: The protocol should be distributed. It should not be dependent on a centralized controlling node. This is the case even for stationary networks. The difference is that the nodes in an ad-hoc network can enter or leave the network very easily and because of mobility the network can be partitioned.

Loop free: To improve the overall performance, the routing protocol should guarantee that the routes supplied are loop free. This avoids any waste of bandwidth or CPU consumption.

Demand based operation: To minimize the control overhead in the network and thus not waste the network resources the protocol should be reactive. This means that the protocol should react only when needed and that the protocol should not periodically broadcast control information.

Unidirectional link support: The radio environment can cause the formation of unidirectional links. Utilization of these links and not only the bi-directional links improves the routing protocol performance.

Security: The radio environment is especially vulnerable to impersonation attacks so to ensure the wanted behaviour of the routing protocol we need some sort of security measures. Authentication and encryption is the way to go and problem here lies within distributing the keys among the nodes in the ad-hoc network.

Power conservation: The nodes in the ad-hoc network can be laptops and thin clients such as PDA's that are limited in battery power and therefore uses some standby mode to save the power. It is therefore very important that the routing protocol has support for these sleep modes.

Multiple routes: To reduce the number of reactions to topological changes and congestion multiple routes can be used. If one route becomes invalid, it is possible that another stored route could still be valid and thus saving the routing protocol from initiating another route discovery procedure. 
Quality of Service Support: Some sort of Quality of service is necessary to incorporate into the routing protocol. This helps to find what these networks will be used for. It could be for instance real time traffic support. It should be noted that none of the proposed protocols have all these properties, but it is necessary to remember that the protocols are still under development and are probably extended with more functionality.

\section{GENERAL CLASSIFICATION FOR ROUTING PROTOCOLS}

There are number of routing protocols currently available in adhoc networks. There is a need for a general technique to classify protocols available. Traditionally classification was done by dividing protocols to table driven and to source initiated[3]. Table Driven routing protocols attempts to maintain consistent up to date routing information for each and every node in the network. These protocols require to maintain a consistent view. The area in which they differ are the number of necessary routing related tables and the methods by which changes in network structure are broadcast.

A very different approach from table driven routing scheme is source initiated routing. This type of routing creates routes only when needed by the source node. When a node needs a route to a destination, it initiates a route discovery process with in the network. This process is completed once route is found or all possible route permutations has been established, it is maintained by a route maintenance procedure until either the destination becomes inaccessible along every path from the source or until the route is no longer required. An efficient classification was introduced by Feeney [9]. This classification is based on to divide protocols according to following criteria, reflecting fundamental design and implementation choices.

- Communication model. What is the wireless communication model? Multi-or single channel?

- Structure. Are all nodes treated uniformly? How are distinguished nodes selected? Is the addressing hierarchical or flat?

- State Information. Is network-scale topology information obtained at each node?

- Scheduling. Is route information continually maintained for each destination?

This model does not care for if a protocol is unicast , multicast or geocast. Also it does not deal with how links are measures. In order to overcome this, Finnish Defence force naval academy modified the model by introducing Type cast routing and Cost function routing.

There are no measures taken to classify the protocols according to power consumption and awareness in routing protocols. In order to overcome this, we add power aware routing to this model.

\subsection{Communication Model:}

The routing protocols presently available can be categorised according to communication model to protocols that are designed for multi-channel or single channel. The example of multichannel protocol is clustered Gateway switched routing (CGSR).Single channel presumes one shared media to be used.

\subsection{Structure:}

Routing protocols can be categorised according to structure as:

Uniform routing: In uniform routing, all nodes acts as same manner as that of other nodes. Sending and receiving messages are control in same way by each and every node. No hierarchy is present in network.

Non-Uniform routing: In this type, there is an effort for the limiting of routing complexity by reducing the number of nodes participating in routing computation.

\subsection{State of Information:}

Protocols can be divided according to state of information obtained at each node as under:

Topology Based routing: This maintains a large scale topology information for each node to participating in topology based protocols. The topology based protocols follows the basic principle of link state protocols.

\subsection{Destination Based routing:}

This does not maintain a large scale topology information but maintains topology information needed to know the nearest neighbours. i.e, Each node exchanges its distance estimates for all network nodes with each of its immediate neighbours.

\section{SCHEDULING}

Depending on when the route is computed, routing protocols can be divided into two categories.

Proactive: Also known as table - driven routing .In this method, the route to all destination are computed a prior i. In order to compute routes in advance, nodes need to store the entire or partial information about link and network topology. To keep the information up to date , nodes need to update their information periodically or whenever the link state or network topology changes. There is no latency.

Re-Active: Also known as on-demand routing. In this method, the route to a destination may not exists in advance and it is computed only when the route discovery process usually initiates the route requested. Once a route has been established, it is inaccessible or until is no longer used or expired.

\section{TYPE CAST ROUTING}

Another type of classification can be done via, type caste property. I.e, Whether they use

$$
\begin{array}{ll}
\text { - } & \text { UniCast } \\
\text { - } & \text { GeoCast } \\
\text { - } & \text { MultiCast }
\end{array}
$$

Unicast: Unicast forwarding means one to one communication. I.e, One source transmits data packets to a single destination.

GeoCast: The main aim of Geocast is to deliver the data to a group of nodes situated inside a specified geographical area.

Multi Cast: Multicast means one to many I.e, when a node needs to send same data to multiple destinations. 


\section{POWER AWARE ROUTING:}

This classification of protocols is based on the consumption of energy during transmission. I.e, Energy required to transmit a signal is approximately proportional to $\mathrm{dX}$, where $\mathrm{d}$ is distance and is the attenuation factor or path loss exponent, which depends on transmission medium. When $\mathrm{X}=2$, which is optimal case, Transmitting a signal half of the distance required one fourth of energy and if a node is in the middle, will spend another fourth of energy for the second half. Thus data will transmit for half of the energy than through direct transmission.

\section{OVERVIEW OF ROUTING PROTOCOLS ACCORDING TO CLASSIFICATION CRITERIA}

The overview of the protocols will be done according to classification criteria discussed above. We will discuss one protocols from every criteria and also mention other protocols that comes under that category.

\subsection{Single Channel Routing :}

\section{Optimized Link State Routing Protocol (OSLR)} OLSR is a comparatively newer proactive routing protocol [15]. It is an adaptation of conventional linkstate routing in which each node tries to maintain information about the network topology. Each node determines the link costs to each of its neighbours by broadcasting HELLO messages periodically. Whenever there is a change in the link costs, the node broadcasts this information to all other nodes. In classical link-state algorithms, this is done by each node flooding the whole network with update packets containing updated link costs. Nodes use this information to apply a shortest path algorithm (such as Dijkstra's shortest path algorithm [8]) to determine the best route to a specific destination. OLSR optimizes the link-state protocol in two ways. First, it reduces the size of the update packets sent during the broadcasts by including only a subset of links to its neighbours. These are the links to a select set of neighbours known as the multipoint relays (MPR). The set of MPRs of a node consist of the minimum set of one hop neighbours of that node so that the node can reach all of its two hop neighbours by using these nodes as relay points. Each node computes its MPR set from the exchange of neighbourhood information with all its neighbours. Second, instead of every neighbour broadcasting the update packets sent out by a node, only the MPR nodes participate in broadcasting of these packets in OLSR. This minimizes the traffic of control packets during flooding. However, the savings of bandwidth achieved using these two techniques come at a cost of propagating incomplete topology information in the network. The updates include only MPR sets and not the sets of all neighbours of the broadcasting nodes. Hence, a shortest path algorithm based on this partial topology information will generate routes containing the MPR nodes only. When the network is dense, i.e. when each node has many neighbours, OLSR will work out to be efficient due to the reduction of control traffic for updates in the network.

Some other protocols of single channel are DSR, GSR, DSDV, WRP, AODV, and ABR.

\subsection{Multi Channel Routing}

Clusterhead Gateway Switch Routing Clusterhead-Gateway Switch Routing (CGSR) [2] is a typical cluster based hierarchical routing. A stable clustering algorithm Least Clusterhead Change (LCC) is used to partition the whole network into clusters and a clusterhead is elected in each cluster. A mobile node that belongs to two or more clusters is a gateway connecting the clusters. Data packets are routed through paths having a format of "Clusterhead - Gateway - Clusterhead - Gateway ..." between any source and destination pairs. CGSR is a distance vector routing algorithm. Two tables, a cluster member table and a DV routing table, are maintained at each mobile node. The cluster member table records the Clusterhead for each node and is broadcast periodically. A node will update its member table upon receiving such a packet. The routing table only maintains one entry for each cluster recording the path to its clusterhead, no matter how many members it has. To route a data packet, current node first looks up the clusterhead of the destination node from the cluster member table. Then, it consults its routing table to find the next hop to that destination cluster and routes the packet towards the destination clusterhead. The destination clusterhead will finally route the packet to the destination node, which is a member of it and can be directly reached. This procedure is demonstrated in Figure 1. The major advantage of CGSR is that it can greatly reduce the routing table size comparing to DV protocols. Only

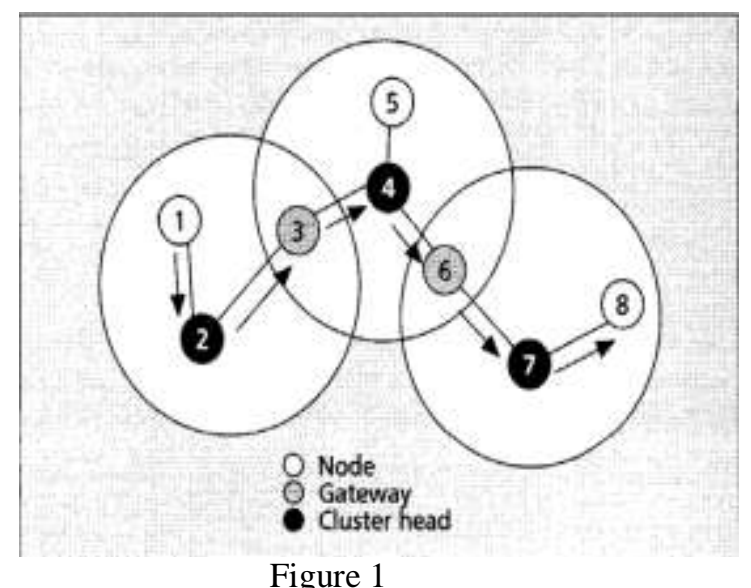

One entry is needed for all nodes in the same cluster. Thus the broadcast packet size of routing table is reduced. These features make a DV routing scale to large network size. Although an additional cluster member table is required at each node, its size only decided by the number of clusters in the network. The drawback of CGSR is the difficulty to maintain the cluster structure in mobile environment. The LCC clustering algorithm introduces additional overhead and complexity in the formation and maintenance of clusters.

Some other protocols of Multi channel are TLR, TRR and TORA. 


\subsection{Uniform Routing:}

\section{Dynamic Source Routing (DSR):}

DSR is a reactive uniform routing protocol that uses a concept called source routing [5]. Each node maintains a route cache where it lists the complete routes to all destinations for which the routes are known. A source node includes the route to be followed by a data packet in its header. Routes are discovered on demand by a process known as route discovery. When a node does not have a route cache entry for the destination to which it needs to send a data packet, it initiates a route discovery by broadcasting a route REQUEST or QUERY message seeking a route to the destination. The REQUEST packet contains the identities of the source and the desired destination. Any node that

receives a REQUEST packet first checks its route cache for an existing entry to the desired destination. If it does not have such an entry, the node adds its identity to the header of the REQUEST packet and transmits it. Eventually, the REQUEST packet will flood the entire network by traversing to all the nodes tracing all possible paths. When a REQUEST packet reaches the destination, or a node that has a known route to the destination, a REPLY is sent back to the source following the same route that was traversed by that REQUEST packet in the reverse direction. This is done by simply copying the sequence of node identities obtained from the header of REQUEST packet. The REPLY packet contains the entire route to the destination, which is recorded in the source node's route cache. When an existing route breaks, it is detected by the failure of forwarding data packets on the route. Such a failure is observed by the absence of the link layer acknowledgement expected by the node where the link failure has occurred. On detecting the link failure, the node sends this information back an ERROR packet to the source. All nodes that receive the ERROR packet, including the source, delete all existing routes from their route caches that contain the specified link. If a route is still needed, a fresh route discovery is initiated.

Some other uniform routing protocols are OSR, DSDV, WRP,AODV,TORA and ABR.

\subsection{Non-Uniform Routing:}

\section{Fisheye State Routing (FSR):-}

Fisheye State Routing (FSR) [14] is an enhancement of GSR. The large size of update messages in GSR dissipates a substantial amount of network bandwidth.

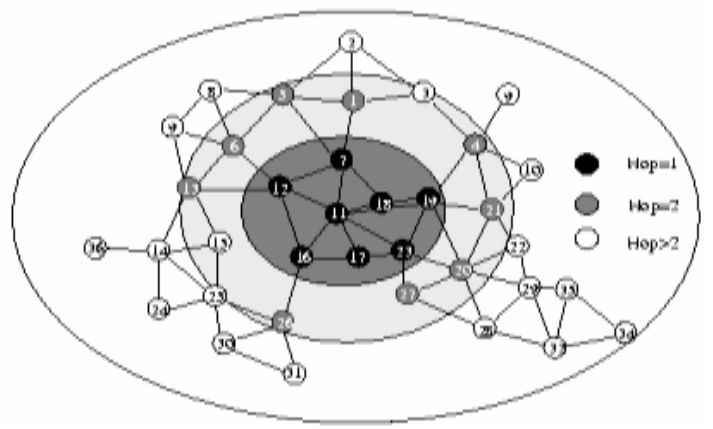

Figure 3

In order to overcome this problem, FSR will use a method where each updated messages would not includes information about all nodes. As an alternative, it swaps information about neighbouring nodes regularly than it does about farther nodes, thus reducing the update message size. In this way, each node gets accurate information about near neighbours and accuracy of information decreases as the distance from the node increases. Even though a node does not have accurate information about distant nodes, the packets are routed correctly because the route information becomes more and more accurate as the packet moves closer to the destination.

Some other protocols are ZRP, OSLR, CEDAR, CBRP, CGSR, DST, HSR and LANMAR.

7.5 Topology Based Routing:

\section{Global Source Routing(GSR)-}

Global State Routing (GSR) [1] is almost the same as DSDV, because it has the idea of link state routing but it makes a progress by decreasing the flooding of routing messages. In this algorithm, each node maintains a neighbour list, a topology table, a next hop table and a distance table.

-The neighbour list of a node includes the list of its neighbours (all nodes that can be heard by it).

- The link state information for each destination is maintained in the topology table together with the timestamp of the information.

- The next hop table includes the next hop to which the packets for each destination must be dispatched.

- The distance table contains the shortest distance to each destination node. The routing messages will be created on a link change as in all link state protocols. Whenever it accepts a routing message, the node updates its topology table if the sequence number of the message is later than the sequence number stored in the table and it then reconstructs its routing table and broadcasts the information to its neighbours.

Some other protocols are DSR,DREAM and GEDIR.

\subsection{Destination Based Routing: \\ Ad-Hoc On-Demand Routing}

AODV is a modification of the DSDV algorithm. When a source node desires to establish a communication session, it initiates a path-discovery process to locate the other node. The source node broadcasts a RREQ packet with its IP address, Broadcast ID (BrID), and the sequence number of the source and destination[4]. While, the BrID and the IP address is used to uniquely identify each request, the sequence numbers are used to determine the timeliness of each packet. Receiving nodes set the backward pointer to the source and generates a RREP unicast packet if it is the destination or contains a route to the destination with a sequence number greater than or equal to the destination sequence number contained in the original RREQ. As the RREP is routed back to the source, forward pointers are setup by the intermediate nodes in their routing tables. The deletion of a route would occur if an entry was not used within a specified lifetime. Link failures are propagated by a RREP message with infinite metric to the source node where route discovery would again occur. An optional feature of AODV is the use of hello messages to maintain the connectivity of neighboring nodes. The hello protocol 
yields a greater knowledge of the network and can improve the route discovery process. The authors of AODV maintain that it is a pure distributed on-demand approach that minimizes routing table information. However, this also means, that more route requests are generated. This compounded with the periodic 'hellos' can increase routing overhead. The advantage of AODV is its use of destination numbers and replies to the first arriving RREQ implies that AODV favours the least congested route instead of the shortest route. Furthermore, another advantage is that the drafted standard supports both unicast and multicast packet transmissions.

Some other protocols are WRP,DSDV, ABR and TORA.

\subsection{Proactive Routing:}

Destination-Sequenced Distance-Vector Routing

- The Destination-Sequenced Distance-Vector Routing protocol (DSDV) described in [3] is a table-driven algorithm based on the classical Bellman-Ford routing mechanism [12]. The improvements made to the Bellman-Ford algorithm include freedom from loops in routing tables. Every mobile node in the network maintains a routing table in which all of the possible destinations within the network and the number of hops to each destination are recorded. Each entry is marked with a sequence number assigned by the destination node. The sequence numbers enable the mobile nodes to distinguish stale routes from new ones, thereby avoiding the formation of routing loops. Routing table updates are periodically transmitted throughout the network in order to maintain table consistency. To help alleviate the potentially large amount of network traffic that such updates can generate, route updates can employ two possible types of packets. The first is known as a full dump. This type of packet carries all available routing information and can require multiple network protocol data units (NPDUs). During periods of occasional movement, these packets are transmitted infrequently. Smaller incremental packets are used to relay only that information which has changed since the last full dump. Each of these broadcasts should fit into a standard-size NPDU, thereby decreasing the amount of traffic generated. The mobile nodes maintain an additional table where they store the data sent in the incremental routing information packets. New route broadcasts contain the address of the destination, the number of hops to reach the destination, the sequence number of the information received regarding the destination, as well as a new sequence number unique to the broadcast [3]. The route labelled with the most recent sequence number is always used. In the event that two updates have the same sequence number, the route with the smaller metric is used in order to optimize (shorten) the path. Mobiles also keep track of the settling time of routes, or the weighted average time that routes to a destination will fluctuate before the route with the best metric is received (see [3]). By delaying the broadcast of a routing update by the length of the settling time, mobiles can reduce network traffic and optimize routes by eliminating those broadcasts that would occur if a better route was discovered in the very near future.

Some other protocols are WRP, FSR, OSLR, CGSR and TBRPF.

\subsection{Reactive Routing: \\ TORA -Temporally Ordered Routing Algorithm} TORA is a link reversal routing (LRR) algorithm and was introduced by Park and Corson in 1993. It evolved from LMR and combines also features from GafniBertzekas in a unique single-pass strategy. In this context "single pass" means, that by processing a single event, all route maintenance tasks (erroneous route deletion, search and establish new routes) can be combined. TORA is a distributed protocol designed to be highly adaptive so it can operate in a dynamic network. For a given destination, TORA uses a somewhat arbitrary 'height' parameter to determine the direction of a link between any two nodes. As a consequence of this multiple routes are often present for a given destination, but none of them are necessarily the shortest route. For a node to initiate a route, it broadcasts a Query to its neighbours. This is rebroadcast through the network until it reaches the destination, or a node that has a route to the destination. This node replies with an Update that contains its height with respect to the destination, which is propagated back to the sender. Each node receiving the Update sets its own height to one greater than that of the neighbour that sent it. This forms a series of directed links from the sender to the destination in order of decreasing height. When a node discovers link failure, it sets its own height higher than that of its neighbours, and issues an Update to that effect reversing the direction of the link between them. If it finds that it has no downstream neighbours, the destination is presumed lost, and it issues a Clear packet to remove the invalid links from the rest of the network.

Some other protocols are AODV, DSR and ABR.

\subsection{MultiCast Routing:}

\section{Distance Vector Multicast Routing Protocol(DVMRP)}

DVMRP supports the formation of source-based trees i.e. the tree is rooted at the network which contains the source sending the data. The routers maintain a consistent view of the underlying unicast topology by periodic exchange of route reports which contain unicast routing tables. All downstream routers convey to the upstream router that they will use the upstream router as the next hop while unicasting data towards a particular source. As a result of these, the upstream router maintains a table of pairs i.e. the source network and a list of dependant downstream routers. This technique is called Poison Reverse Technique. For example, consider the topology of Figure 4. The routers R2, R3 and R4 tell the router $\mathrm{R} 1$ that while unicasting data towards the source (i.e. network N1) they will use it as the next hop towards it. The router $\mathrm{R} 1$ maintains this information in a table. The formation of trees is data-driven. A router starts building the router entries for any (source, group) pair only after it receives the first datagram belonging to this pair. In the beginning, the source starts forwarding the data on all outgoing links without any knowledge of the topology of the Each router on receiving the packet belonging to a particular (source, group) pair performs a check whether the packet has arrived on the same interface which it uses to forward unicast data towards that source. For example, in the topology of Figure 4 router R3 


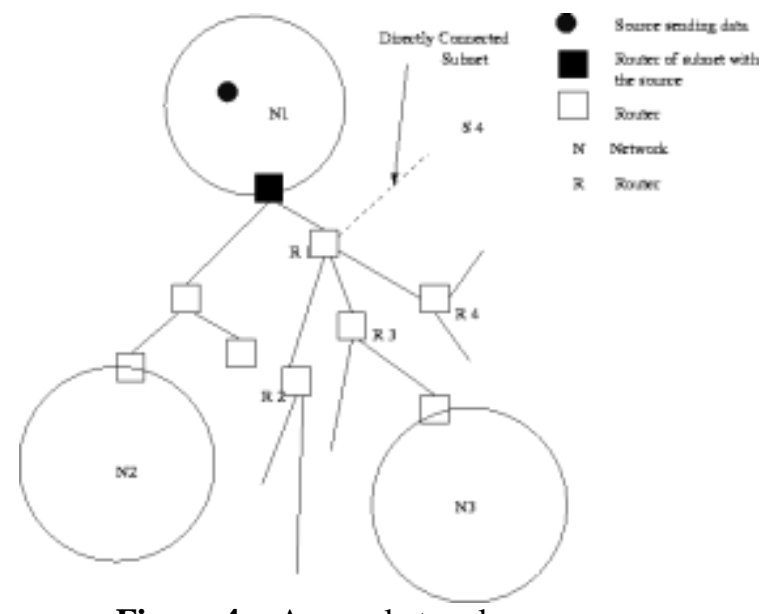

Figure 4: A sample topology

on receiving a data packet from $\mathrm{S} 1$ checks whether it receives it on the interface on which it is connected to $\mathrm{R} 1$ (the first router towards the source S1). This check is called Reverse Path Forwarding. If the check succeeds, the packet is forwarded to a list of interfaces obtained through Poison Reverse Technique, and those which have directly attached networks with group members. The router also creates an entry in its internal tables. A typical entry consists of the source network address, the multicast group address, the incoming interface identifier, the forwarding or outgoing interface list and a few timers such as the age timer for the entry, the prune timer etc. This way the datagram is flooded to the entire network. Now, the leaf routers i.e. those routers which have no downstream multicast routers and no directly attached networks with group members, on receiving the initial datagram sends a prune message for this (source, group) pair upstream. On receiving a prune message, the interface-id on which this prune message is received is deleted from the router entry. If there are no directly attached networks or downstream dependant routers for this (source, group) pair, then this router can also be pruned. It will forward the prune message upstream. This way the branches of the tree are pruned off. Pruning has been made a necessity in DVMRP employed over MBONE. Again consider the topology of Figure 4. Suppose that R3 and R4 send a prune for a (source, group) pair towards R1. On receiving these prunes R1 checks whether any members are present on the directly attached network (i.e. S4) or on any downstream dependant router (i.e. R2). If no member is present it itself sends a prune upstream. There is a timer associated with prune state in a router. After the timer expires, the prune information is deleted, and any future packets will be forwarded on all outgoing interfaces. This is done to account for the fact that group membership is dynamic and the members can join the group at any time. On receiving these datagrams, the downstream router has to send a prune message again in order to disable the branch. Thus there is an alternate period of flooding and pruning. When a new network wants to add itself to the tree, the corresponding router would not send a prune message next time. However, if the prune timer is large, this will delay the addition. To join the group immediately, there is a provision of graft message. This message is sent to the upstream router, towards the source. Each upstream router acknowledges the graft message and then further sends a graft upstream if it itself is in the prune state for that particular (source, group) pair. This way the branches are added to the tree.

Some other are DVMRP, CAMP, ODMRP.

\subsection{Geo-Cast Routing: GeoGRID:}

The basic idea of GRID is to partition the network into logical grids. In each grid one host close to the grid center is elected to be the responsible node for propagating geocast packets to neighboring grids. These hosts are called gateways. Geocast packets are sent in a grid-by-grid manner through their gateways. Thus, gateways are responsible for forwarding geocast packets to neighboring grids, which decreases message overhead by relieving non-gateways from packet flooding. The assumption is that grid sizes are constructed such that a gateway is able to communicate with at least one or more other gateways without relaying. There are two suggestions in [17] on how to send geocast packets by GeoGRID:

\section{- $\quad$ Flooding-Based GeoGRID Ticket-Based GeoGRID.}

Flooding Based GoeGRID: It rebroadcasts the packet to its neighbour gateways provided that the packet is not a duplicate of a packet that was already broadcast. Inside the destination region a mobile node delivers a received packet to its upper application layers.

Ticket Based GoeGRID: In this second scheme again a gateway within the forwarding region forwards geocast packets, but only a limited number of gateways will do this. To limit the number of gateways, a gateway forwarding a packet sends it to at most three neighbours rather than to every neighbour and the initial sender limits the overall fan-out of the flooding by specifying a number of tickets. The idea is that each ticket is responsible for carrying one copy of the geocast packet to the destination region. Thus, by selecting a certain number of tickets the initial sender not only determines the overhead of geocast delivery but also the success probability of delivery.

Some other protocols are GeoTORA and LBM.

\subsection{Power Aware Routing: \\ PARO:}

PARO[10], A power aware routing technique for wireless ad hoc networks where all nodes are located within the maximum transmission range of each other. PARO uses a packet forwarding technique where immediate nodes can elect to be redirectors on behalf of source-destination pairs with the goal of reducing the overall transmission power needed to deliver packets in the network, thus, increasing the operational lifetime of networked devices. Prior to transmitting a packet, a node updates its packet header to indicate the power required to transmit the packet. A node overhearing another node's transmission can then use this information plus, a localized measure of the received power, to compute (using a propagation model) the minimum transmission power necessary to reach the overheard node. In this simple manner, nodes can learn the minimum transmission power toward neighboring nodes. PARO 
does not, however, maintain routes to other nodes in the network in advance but discovers them on a per-node ondemand basis. This approach has the benefit that signalling packets, if any, are transmitted only when an unknown route to another node is required prior to data transmission, thus reducing the overall power consumption in the network.

Some other protocols are EADSR, DSRPA and ISAHIAH.

\section{CONCLUSION}

In this article we provide descriptions of several routing schemes proposed for ad hoc wireless networks. We also provide a classification of these schemes according to the routing strategy. The presented classification model of routing protocols is a meaningful attempt to clarify the vast field of adhoc routing protocols. It is so because it tries to reveal the main design and implementation principles behind protocols. The classification is a little bit complicated and it is not always an easy task to classify a protocol according to that taxonomy, but the meaning of classifying is try to get some rough basis for protocol's performance evaluation. In future we will study category wise performance analysis of routing protocols.

\section{REFERENCES}

1. A.Iwata, C.-C. Chiang, G. Pei, M. Gerla, and T.-W. Chen, "Scalable Routing Strategies for Ad Hoc Wireless Networks" IEEE Journal on Selected Areas in Communications, Special Issue on Ad-Hoc Networks, Aug. 1999, pp.1369- 79.

2. C. -C. Chiang and M. Gerla, "Routing and Multicast in Multihop, Mobile Wireless Networks," n Proceedings of IEEE ICUPC'97, San Diego, CA, Oct. 1997.

3. C. E. Perkins and P. Bhagwat, "Highly Dynamic Destination-Sequenced Distance-Vector Routing (DSDV) for Mobile Computers," Comp. Commun. Rev., Oct. 1994, pp. 234-44.

4. Charles Perkins and Elizabeth Royer. Ad hoc on demand distance vector (AODV) routing.http://www.ietf.org/internet-drafts/draft-ietfmanet-aodv-02.txt, November 1998. IETF Internet Draft

5. D. Johnson and D. Maltz. Dynamic source routing in ad hoc wireless networks. In T. Imielinski and H. Korth, editors, Mobile computing. Kluwer Academic, 1996
6. E.M. Royer, C-K. Toh, A Review of Current Routing Protocols for Ad-Hoc Mobile Wireless Networks, IEEE Personal Communications Magazine, April 1999, pp.46-55. http://www.cs.ucsb.edu/ eroyer/ publications.html

7. E.L. Madruga, J.J. Garcia -Luna-Aceves. Scalable Multicasting: The Core-Assisted Mesh Protocol. 1999.

http://www.ee.surrey.ac.uk/Personal/G.Aggelou/PA PERS/madruga.monet99.pdf

8. E. W. Dijkstra. A note on two problems in connection with graphs. Numerical Mathematics, 1:269-271, Oct. 1959

9. Freebersyser, J.A., and Barry Leiner, 2001. A DoD perspective on mobile ad hoc networks, in: Charles E. Perkins (Ed.), Ad Hoc Networking, Addison Wesley, Reading, MA, pp. 29-51.

10. JAVIER GOMEZ and ANDREW T.CAMPBELL "PARO: Supporting Dynamic Power Controlled Routing in Wireless Ad Hoc Networks" Kluwer Academic Publishers. Wireless Networks 9, 443460, 2003.

11. Kuosmanen, P., 2002. Classification of ad hoc routing protocols, Finnish Defence forces, Naval Academy, Finland. available from http://keskus.hut.fi/opetus/s38030 /k02/Papers/12Petteri.pdf $>$.

12. L. R. Ford Jr. and D. R. Fulkerson, Flows in Networks, Princeton Univ.Press, 1962

13. M. R. Perlman and Z. J. Haas. Determining the optimal configuration for the zone routing rotocol. IEEE Journal on Selected Areas in Communications, 17(8):1395-1414, August 1999.

14. Mingliang Jiang, Jinyang Li, Y.C. Tay, "Cluster Based Routing Protocol" August 1999IETFDraft.http://www.ietf.org/internetdrafts/draft-ietf-manetcbrp-spec-01 .txt

15. P. Jacquet et al. Optimized link state routing protocol. draft-ietf-manet-olsr-05.txt, 2000. IETF Internet Draft

16. S. Liu. QoS routing in ad hoc wireless networks. IEEE Journal on Selected Areas in Communications, 17(8):1426-1438, August 1999.

17. W.-H. Liao, Y.-C. Tseng, K.-L. Lo, and J.-P. Sheu. Geogrid: A geocasting protocol for mobile ad hoc networks based on grid. Journal of Internet Technology, 1(2):23-32, 2000. 